\title{
UNITARY ONE-PARAMETER GROUPS WITH FINITE SPEED OF PROPAGATION
}

\author{
E. C. SVENDSEN
}

\begin{abstract}
Suppose that $\xi$ is a Hermitian vector bundle over a Riemannian manifold and that $U$ is a one-parameter group of linear operators on the set of smooth sections of $\xi$ with compact support. We prove that if $U$ satisfies a smoothness condition, is unitary, and propagates initial data with finite speed, then it can be constructed from the solutions of a first-order symmetric hyperbolic system of partial differential equations.
\end{abstract}

Suppose that $\xi$ is a Hermitian vector bundle over a Riemannian manifold $M$. In [2] Chernoff studied first-order symmetric hyperbolic systems of partial differential equations associated with $\xi$. He proved that if $M$ is complete and the coefficients of such a system are time-independent, smooth, and sufficiently small, then the solutions of the system can be used to construct a one-parameter group of linear operators on the set of smooth sections of $\xi$ with compact support. He also proved that under these conditions this one-parameter group has three properties: it satisfies a smoothness condition, is unitary, and propagates initial data with finite speed.

In this paper we prove a converse to Chernoff's results: any one-parameter group of linear operators on the set of smooth sections of $\xi$ with compact support with these three properties can be constructed from the solutions of a first-order symmetric hyperbolic system belonging to a natural class of such systems. If $M$ is complete, this result, together with Chernoff's results, characterizes the systems belonging to this class.

We will use local techniques to prove our result. Fourier transform techniques (such as those developed in [3] by Gel'fand and Šilov) can be used to prove results like ours for translation-invariant one-parameter groups on spaces of functions on $R^{n}$ or other Lie groups; in [1] Berman used such techniques to characterize certain translation-invariant second-order wave equations. (Our techniques can also be used to characterize such higher-order equations.)

Before stating our result more precisely, we give a number of preliminaries:

Suppose that $c$ is a nonnegative real number (the upper limit on the speed of propagation). Also, suppose that $M$ is a $C^{\infty}$ manifold with a $C^{\infty}$ Riemannian metric $\langle-,-\rangle_{M}$ and that $\xi$ is a complex $C^{\infty}$ vector bundle over $M$ with a $C^{\infty}$ Hermitian structure $\langle-,-\rangle_{\xi}$ (inner product on each fiber $\xi_{x}$ of $\xi$ ).

Received by the editors November 11, 1980 and, in revised form, May 1, 1981.

1980 Mathematics Subject Classification. Primary 35L45, 58G17; Secondary 47D05.

Key words and phrases. Symmetric hyperbolic system, finite speed of propagation, initial-value problem, unitary one-parameter group, Hermitian vector bundle.

(c) 1982 American Mathematical Society 0002-9939/81/0000-1021/\$02.25 
Let $C_{0}^{\infty}(\xi)$ denote the set of $C^{\infty}$ sections of $\xi$ with compact support, and let $\langle-,-\rangle$ denote the inner product on $C_{0}^{\infty}(\xi)$ defined by

$$
\langle f, g\rangle=\int_{M}\langle f, g\rangle_{\xi} d V,
$$

where $d V$ is the measure associated with the Riemannian metric.

We now define the class $\mathcal{Q}(c)$ of one-parameter groups appearing in our result: Suppose that $U$ is a one-parameter group of linear operators on $C_{0}^{\infty}(\xi)$. If $f$ is in $C_{0}^{\infty}(\xi)$, let $U f$ be the function from $M \times R$ into $\xi$ defined by

$$
U f(x, t)=U_{\imath} f(x) \text {. }
$$

Say that $U$ is smooth if $U f$ is $C^{\infty}$ for all $f$ in $C_{0}^{\infty}(\xi)$. And say that $U$ propagates initial data with speed $c$ or less if, for all $f$ in $C_{0}^{\infty}(\xi)$ and $t$ in $R$, the distance between the support of $f$ and any point in the support of $U_{\downarrow} f$ is less than or equal to $c|t|$. Let $U(c)$ denote the set of smooth unitary one-parameter groups of linear operators on $C_{0}^{\infty}(\xi)$ that propagate initial data with speed $c$ or less.

If $U$ is a smooth one-parameter group of linear operators on $C_{0}^{\infty}(\xi)$, let $d U$, the infinitesimal generator of $U$, be the linear operator on $C_{0}^{\infty}(\xi)$ defined by

$$
d U f(x)=\frac{\partial U f}{\partial t}(x, 0) .
$$

Our result is in terms of the operators $d U$; we now define the class $\mathcal{L}(c)$ of such operators appearing in it: Suppose that $L$ is a zeroth- or first-order linear differential operator on $C_{0}^{\infty}(\xi)$. In [2] Chernoff defined the speed of propagation $c_{L}(x)$ of $L$ at a point $x$ in $M$ as follows:

$$
c_{L}(x)=\sup \left\{\left\|\sigma_{L}(u, x)\right\|_{\mathrm{op}}: u \in T_{x}^{*} M,\|u\|_{\mathrm{cs}}=1\right\}
$$

where $\sigma_{L}$ is the symbol of $L,\|-\|_{o p}$ is the operator norm, and $\|-\|_{c s}$ is the Riemannian norm on the cotangent space $T_{x}^{*} M$. Let $\mathcal{L}(c)$ denote the set of formally skew-adjoint zeroth- or first-order linear differential operators on $C_{0}^{\infty}(\xi)$ such that $c_{L}(x)<c$ for all $x$ in $M$.

We can now state our result:

THEOREM 1. $d(\mathcal{Q}(c)) \subset \mathcal{E}(c)$.

This result implies that if $U$ is in $\mathscr{U}(c)$, then there is a first-order symmetric hyperbolic system

$$
\partial \tilde{f} / \partial t=L \tilde{f}
$$

with $L$ in $\mathcal{L}(c)$ such that if $f$ is in $C_{0}^{\infty}(\xi)$, then $U f$ is the unique $C^{\infty}$ solution of (1) satisfying the initial condition $\tilde{f}(x, 0)=f(x) .(L=d U$, of course.)

In [2] Chernoff obtained results that imply that if $M$ is complete, then $d(\mathcal{Q}(c)) \supset$ $\mathcal{L}(c)$. Thus we have the following

COROLlaRY. If $M$ is complete, then $d(U(c))=\mathcal{L}(c)$.

If $U_{1}$ and $U_{2}$ are in $\mathscr{U}(c)$ and $d U_{1}=d U_{2}$, then $U_{1}=U_{2}$ (as one can easily show); this fact and the corollary imply that if $M$ is complete, then the map $U \rightarrow d U$ establishes a one-to-one correspondence between $\mathcal{U}(c)$ and $\mathcal{L}(c)$. 
Before proving Theorem 1, we discuss the nature of $d U$ when $U$ is not in $U(c)$. Suppose that $U$ is a smooth one-parameter group of linear operators on $C_{0}^{\infty}(\xi)$ (so that $d U$ is defined). If $U$ propagates initial data with speed $c$ or less for some $c$, then $d U$ is a differential operator, as our proof of Theorem 1 will show. However, if $U$ does not propagate initial data with speed $c$ or less for any $c$, then $d U$ might not be a differential operator. For example, suppose that $M$ is $R$ with its usual Riemannian metric, that $\xi$ is the product vector bundle $R \times C^{2}$ over $R$ with its usual Hermitian structure, and that $a$ is a nonzero real number; now define $U$ by

$$
U_{t}\left(f_{1}, f_{2}\right)(x)=\left(f_{1}(x), f_{2}(x)+t f_{1}(x+a)\right) .
$$

Then $U$ does not propagate initial data with speed $c$ or less for any $c$, and $d U$ is not a differential operator. And even if $U$ does propagate initial data with speed $c$ or less for some $c$ (so that $d U$ is a differential operator), $d U$ might not have order zero or one. For example, suppose that $M$ and $\xi$ are as above and that $k$ is an integer greater than one; now define $U$ by

$$
U_{t}\left(f_{1}, f_{2}\right)(x)=\left(f_{1}(x), f_{2}(x)+t f_{1}^{(k)}(x)\right) .
$$

Then $U$ propagates initial data with speed zero, but $d U$ has order greater than one ( $k$, in fact). ( $U$ is not unitary, of course.)

Proof of Theorem 1. Suppose that $U$ is in $U(c)$, and let $L=d U$. Since $U$ propagates initial data with speed $c$ or less, $L$ does not increase supports. According to a theorem of Peetre [5], $L$ is therefore a differential operator. The formal skew-adjointness of $L$ can be proved in the usual way (by differentiating the equation expressing the unitarity of $U$ with respect to $t$ and setting $t$ equal to zero).

Our proof uses a domain-of-dependence inequality involving integrals over closed balls $B(a, \tilde{r})$ with center $a$ and radius $\tilde{r}$. Suppose that $r>0$ and that $f$ is in $C_{0}^{\infty}(\xi)$, and let

$$
I(t)=\int_{B(a, r+c|t|)}\left\|U_{t}\right\|_{\xi}^{2} d V
$$

where $\|-\|_{\xi}$ is the norm associated with $\langle-,-\rangle_{\xi}$; the inequality we need is

$$
I(t) \geqslant I(0) \text {. }
$$

This can be proved using a sequence $\varphi_{k}$ of $C^{\infty}$ cut-off functions. More specifically, suppose that $\varphi_{k}$ is identically equal to 1 on $B(a, r+c|t|)$ and is identically equal to 0 on $B\left(a, r+c|t|+\frac{1}{k}\right)$. Then $\varphi_{k} U_{t} f$ and $U_{t} f$ agree on $B(a, r+c|t|)$, and

$$
\int_{M}\left\|\varphi_{k} U_{t}\right\|_{\xi}^{2} d V \rightarrow I(t)
$$

Since $U$ propagates initial data with finite speed or less, $U_{-t}\left(\varphi_{k} U_{\downarrow} f\right)$ and $U_{-t}\left(U_{t} f\right)=$ $f$ agree on $B(a, r)$. Therefore

$$
\int_{M}\left\|U_{-t}\left(\varphi_{k} U_{\downarrow} f\right)\right\|_{\xi}^{2} d V \geqslant I(0)
$$

(2) follows from (3), (4), and unitarity. 
Suppose that $B(a, r)$ is contained in a normal neighborhood of $a[4, \mathrm{p}$. 33]. Then $I^{\prime}(0+)$ exists and is given by

$$
I^{\prime}(0+)=c \int_{S(a, r)}\|f\|_{\xi}^{2} d S+\int_{B(a, r)}\left(\langle L f, f\rangle_{\xi}+\langle f, L f\rangle_{\xi}\right) d V
$$

where $S(a, r)$ is the sphere with center $a$ and radius $r$, and $d S$ is the measure on $S(a, r)$ associated with the Riemannian metric on it induced by $\langle-,-\rangle_{M}$. (2) implies that $I^{\prime}(0+) \geqslant 0$. Therefore

$$
-\int_{B(a, r)}\left(\langle L f, f\rangle_{\xi}+\langle f, L f\rangle_{\xi}\right) d V<c \int_{S(a, r)}\|f\|_{\xi}^{2} d S .
$$

We will prove that (5) is violated if $L$ is not in $\mathcal{L}(c)$. First we write (5) in local coordinates. Suppose that $b$ is on $S(a, r)$ and that $\Omega$ is a neighborhood of $b$. Also, suppose that $x_{1}, \ldots, x_{n}$ are local coordinates for $\Omega$, with $x_{1}$ the distance from $a$. Let $m$ be the order of $L$, and suppose that in these coordinates $L=A_{1} D_{1}^{m}+\ldots$, where the dotted terms contain only lower powers of $D_{1}$. (Given $b$, we can always choose $a, r, \Omega$, and $x_{1}, \ldots, x_{n}$ in such a way that these suppositions are satisfied.) Integration by parts shows that the formal adjoint $L^{\dagger}$ of $L$ is given by

$$
L^{\dagger}=(-1)^{m} A_{1}^{\dagger} D_{1}^{m}+\ldots
$$

Note that

$$
A_{1}^{\dagger}=(-1)^{m+1} A_{1}
$$

(because $L^{\dagger}=-L$ ). Suppose that the support of $f$ is contained in $\Omega$. Then in our coordinates (5) becomes

$$
-\int_{x_{1}<r}\left(\left(A_{1} D_{1}^{m} f\right)^{\dagger} f+f^{\dagger}\left(A_{1} D_{1}^{m} f\right)+\ldots\right) d V<c \int_{x_{1}=r}|f|^{2} d S
$$

(Let $g$ be the determinant of the metric tensor; then $d V$ and $d S$ are $g^{1 / 2}$ times Lebesgue measure on $R^{n}$ and $R^{n-1}$, respectively.) Now we integrate the left side of (7) by parts and then use the formal skew-adjointness of $L$; we find that when $m>1$,

$$
-\int_{x_{1}=r}\left(2 \operatorname{Re}\left(f^{\dagger} A_{1} D_{1}^{m-1} f\right)+\ldots\right) d S \leqslant c \int_{x_{1}=r}|f|^{2} d S
$$

(where again the dotted terms contain only lower powers of $D_{1}$ ), and that when $m=1$,

$$
-\int_{x_{1}=r} f^{\dagger} A_{1} f d S \leqslant c \int_{x_{1}=r}|f|^{2} d S
$$

(When $m=0$, the left side of (7) is equal to zero.)

We will use (8) and (9) to complete our proof. First suppose by way of contradiction that $m>1$. Take a point $b$ in $M$ at which the order of $L$ is exactly $m$. Then choose $a, r, \Omega$, and $x_{1}, \ldots, x_{n}$ in such a way that $A_{1}(b) \neq 0$. But then we can construct an $f$ that violates (8). Therefore $m<1$. Next suppose that $m=1$. Take any point $b$ in $M$ and any unit vector $u$ in $T_{b}^{*} M$. Then choose $a, r, \Omega$, and 
$x_{1}, \ldots, x_{n}$ in such a way that the local coordinates of $u$ are $(1,0, \ldots, 0)$. (We can always do this because $x_{1}$ is the distance from $a$.) With these assumptions,

$$
\sigma_{L}(u, b)=A_{1}(b) \text {. }
$$

(6) implies that $A_{1}(b)$ is selfadjoint and hence has only real eigenvalues. The magnitudes of these eigenvalues must be less than or equal to $c$ because otherwise we could construct an $f$ that violates (9). Therefore

$$
\left\|\sigma_{L}(u, b)\right\|_{\mathrm{op}}=\left\|A_{1}(b)\right\|_{\mathrm{op}}<c,
$$

$c_{L}(b)<c$, and $L$ is in $\mathcal{L}(c)$. Finally, suppose that $m=0$. Then $\sigma_{L}$ and hence $c_{L}$ are identically zero, and therefore $L$ is in $\mathcal{E}(c)$.

ACKNOwLedgements. I would like to thank Paul Chernoff for his encouragement and suggestions. I would also like to thank the referee for his suggestions, which helped me simplify my proof.

\section{REFERENCES}

1. S. J. Berman, Wave equations with finite velocity of propagation, Trans. Amer. Math. Soc. 188 (1974), 127-148.

2. P. R. Chernoff, Essential self-adjointness of powers of generators of hyperbolic equations, J. Funct. Anal. 12 (1973), $401-414$.

3. I. M. Gel'fand and G. E. Šilov, Fourier transforms of rapidly increasing functions and questions of the uniqueness of the solution of Cauchy's problem, Amer. Math. Soc. Transl. (2) 5 (1957), 221-274.

4. S. Helgason, Differential geometry and symmetric spaces, Academic Press, New York, 1962.

5. J. Peetre, Rectifications à l'article "Une caractérisation abstraite des opérateurs différentiels", Math. Scand. 8 (1960), 116-120.

Department of Mathematics, OKlahoma State University, Stillwater, OKLahoma 74078 